\title{
Existence for a Higher Order Coupled System of Korteweg-de Vries Equations
}

\author{
Min Liu \\ College of Sciences, University of Shanghai for Science and Technology, Shanghai, China \\ Email: 745052714@qq.com
}

How to cite this paper: Liu, M. (2021) Existence for a Higher Order Coupled System of Korteweg-de Vries Equations. Applied Mathematics, 12, 298-310. https://doi.org/10.4236/am.2021.124021

Received: March 22, 2021

Accepted: April 16, 2021

Published: April 19, 2021

\section{Copyright $\odot 2021$ by author(s) and} Scientific Research Publishing Inc. This work is licensed under the Creative Commons Attribution International License (CC BY 4.0).

http://creativecommons.org/licenses/by/4.0/

\begin{abstract}
Consider the following system of coupled Korteweg-de Vries equations, $\left\{\begin{array}{l}\Delta^{2} u+\lambda_{1} u=u^{2}+\beta u v^{2}, \\ \Delta^{2} v+\lambda_{2} v=v^{2}+\beta u^{2} v\end{array}\right.$ where $u, v \subseteq W^{2,2}, 2 \leq N \leq 7$ and $\lambda_{i}, \beta>0, \beta$ denotes a real coupling parameter. Firstly, we prove the existence of the solutions of a coupled system of Korteweg-de Vries equations using variation approach and minimization techniques on Nehari manifold. Then, we show the multiplicity of the equations by a bifurcation theory which is rare for studying higher order equations.
\end{abstract}

\section{Keywords}

System of Korteweg-de Vries Equations, Normalized Vector Solitary Waves, Variation Approach

\section{Introduction}

It is well known that the form of the coupled nonlinear Korteweg-de Vries equations is as follows

$$
\left\{\begin{array}{l}
\phi_{t}+\phi_{x x x}+P(\phi, \psi)_{x}=0 \\
\psi_{t}+r \psi_{x}+\psi_{x x x}+Q(\phi, \psi)_{x}=0
\end{array}\right.
$$

where $r$ is a real constant, $\phi=\phi(x, t), \psi=\psi(x, t)$ are real-valued functions of $x$ and $t, \beta>0$ is a coupling parameter and $P, Q$ satisfy

$P(\phi, \psi)=H_{u}(\phi, \psi), Q(\phi, \psi)=H_{v}(\phi, \psi)$ for a small function $H$. Model represents the physical problem of describing the strong interaction of two-dimensional long internal gravity waves propagating on neighboring pycnoclines in a stratified fluid. In this paper we consider a special case of (1.1), namely the following system of nonlinear evolution equations: 


$$
\left\{\begin{array}{l}
\phi_{t}+\phi_{x x x}+\left(u v^{2}\right)_{x}=0, \\
\psi_{t}+r \psi_{x}+\psi_{x x x}+\left(u^{2} v\right)_{x}=0,
\end{array}\right.
$$

We always look for solutions of (1.2) of the form

$$
(\phi(x, t), \psi(x, t))=\left(u\left(x-\lambda_{1} t\right), v\left(x-\lambda_{2} t\right)\right),
$$

where $\lambda_{1}, \lambda_{1} \in \mathbb{R}$. Through calculating, if $r=0$ we get

$$
\left\{\begin{array}{l}
-u^{\prime \prime}+\lambda_{1} u=\beta u^{p} v^{p+1}, \\
-v^{\prime \prime}+\lambda_{2} v=\beta u^{p+1} v^{p},
\end{array}\right.
$$

Now we consider it in higher dimensional cases, as follows:

$$
\left\{\begin{array}{l}
\Delta^{2} u+\lambda_{1} u=u^{2}+\beta u v^{2}, \\
\Delta^{2} v+\lambda_{2} v=v^{2}+\beta u^{2} v,
\end{array}\right.
$$

where $u, v \subseteq W^{2,2}, 2 \leq N \leq 7$ and $\lambda_{i}, \beta>0, \beta$ is a coupling parameter.

The system of (1.1) has been analysed many times. For example, see the recently derived model by Gear and Grimshaw [1], considering

$$
H(u, v)=\frac{b_{2}}{6} u^{3}+\frac{1}{6} v^{3}+\frac{a_{2} b_{2}}{2} u^{2} v+\frac{a_{1} b_{2}}{1} u v^{2},
$$

where $a_{1}, a_{2}, b_{1}, b_{2}$ are constants. Moreover, the system (1.2) has been extensively studied in recent years and is also a special case of a general class of nonlinear evolution equations considered in [2] in the inverse scattering context. More properties of the system (1.2) have been proved. Alarcon and Montenegro proved the local and global well-posedness results for the initial-value problem for (1.2) with $r=0, p=1$ in [3] and [4]. Panthee and Scialom improved the well-posedness results obtained in the case when $p=2$ in [2].

As we know, many analyses about higher order equation have been done many years ago including the third and fifth order KdV equation. Firstly, it is already well known that the third order KdV equation describes the evolution of weakly nonlinear and weakly dispersive shallow waves in physical contexts such as plasma, ion-acoustic waves, stratified internal, and atmospheric waves and it has been analysed during the last decades. For the fifth order equation, the results are less than the third. But it has attracted increasing attentions (see [5]-[22]) and is used to model many physical phenomena such as gravity-capillary waves on a shallow layer and magnetosome propagation in plasmas. For example, Baker took the work and published in 1903; Li Xiaofeng proved the existence of solitary wave solutions of fifth order $\mathrm{KdV}$ equations in recent years. Santosh Bhattarai proved the existence of travelling-wave solutions of coupled $\mathrm{KdV}$ equations when it loses the compactness, using the method of concentrate compactness principle of Lions in 2015.

We know that the system of higher order equations is rare. We only can find other similar fourth-order systems studying the interaction of the long and short waves have appeared. P. Lvarez-Caudevilla and E. Colorado researched the coupled nonlinear Schrodinger Equations (1.7) and the system of Schrodinger 
and Korteweg-de Vries Equations (1.8).

$$
\left\{\begin{array}{l}
\Delta^{2} u+\lambda_{1} u=\mu_{1} u^{3}+\beta u v^{2}, \\
\Delta^{2} v+\lambda_{2} v=\mu_{2} v^{3}+\beta u^{2} v,
\end{array}\right.
$$

and

$$
\left\{\begin{array}{l}
\Delta^{2} u+\lambda_{1} u=u^{3}+\beta u v, \\
\Delta^{2} v+\lambda_{2} v=\frac{1}{2}|v|^{2} v+\beta u^{2},
\end{array}\right.
$$

They proved the existence of equations using the variation approach and minimization techniques on Nehari manifold and the multiplicity of the equations by fibering map.

But we know that there is not previous mathematics work analyzing a higher order system as (1.3) and we get the multiplicity of the equations by a bifurcation theory which is not founded in other higher order equations article.

We organize the paper as follows. In Section 2, we introduce the notation, establish the functional framework, define the Nehari manifold and give the main theorem. In Section 3, we construct semi-trivial solutions and show the properties depending on the coupling parameter. Moreover, we devoted to proving the main results of the paper by the variation principle and mountain-pass theorem. In Section 4, using the Crandall-Rabinowitz local bifurcation theory, we show the multiplicity of the ground state solutions.

\section{Preliminaries and Main Theorems}

In $H^{2}\left(\mathbb{R}^{N}\right)$, we define the following equivalent norm and scalar product:

$$
\langle u, v\rangle_{i}:=\int_{\mathbb{R}^{N}}\left(\Delta u \cdot \Delta v+\lambda_{i} u v\right), \quad\|u\|_{i}^{2}:=\langle u, u\rangle_{i}, i=1,2,
$$

Accordingly, the inner product and induced norm on $H^{2} \times H^{2}$ are given by

$$
\begin{aligned}
& \langle(u, v),(\xi, \eta)\rangle=\int_{\mathbb{R}^{N}}\left(\Delta u \cdot \Delta \xi+\Delta v \cdot \Delta \eta+\lambda_{1} u \xi+\lambda_{2} v \eta\right), \\
& \|(u, v)\|^{2}=\|u\|_{1}^{2}+\|u\|_{2}^{2},
\end{aligned}
$$

We define $\mathbb{E}$ the radially symmetric functions in $H^{2}\left(\mathbb{R}^{N}\right)$ and $\mathbb{H}=H \times H$. In addition, we define the energy functional associated with system (1.5) by

$$
\Phi(\mathbf{u})=\frac{1}{2}\|u\|_{1}^{2}+\frac{1}{2}\|v\|_{2}^{2}-\frac{1}{3} \int_{\mathbb{R}^{N}}\left(u^{3}+v^{3}\right)-\frac{1}{2} \beta \int_{\mathbb{R}^{N}} u^{2} v^{2}
$$

and

$$
I_{1}(u)=\frac{1}{2}\|u\|_{1}^{2}-\frac{1}{3} \int_{\mathbb{R}^{N}} u^{3}, \quad I_{2}(v)=\frac{1}{2}\|v\|_{1}^{2}-\frac{1}{3} \int_{\mathbb{R}^{N}} v^{3},
$$

are the energy functionals of the uncoupled equations. Then, we define

$$
\Psi(\mathbf{u})=\Phi^{\prime}(\mathbf{u})[\mathbf{u}]=\|\mathbf{u}\|^{2}-\int_{\mathbb{R}^{N}}\left(u^{3}+v^{3}\right)-2 \beta \int_{\mathbb{R}^{N}} u^{2} v^{2} .
$$

Now, we restrict the Nehari Manifold to the setting, denoting it as

$$
\mathcal{N}=\{\mathbf{u} \in \mathbb{E} \backslash\{(0,0)\}: \Psi(\mathbf{u})=0\} .
$$


Remark 2.1. (see [23] [24] [25])

Let

$$
2^{*}= \begin{cases}\frac{2 N}{N-4}, & \text { if } N>4 \\ \infty, & \text { if } 1 \leq N \leq 4\end{cases}
$$

Then we have the following Sobolev embedding.

$$
H^{2}\left(\mathbb{R}^{N}\right) \hookrightarrow L^{p}\left(\mathbb{R}^{N}\right), \quad \text { for } \begin{cases}2 \leq p \leq 2^{*}, & \text { if } N \neq 4, \\ 2 \leq p<2^{*}, & \text { if } N=4 .\end{cases}
$$

Proposition 2.1. We are going to prove some properties for $\mathcal{N}$ and $\Phi$ on $\mathcal{N}$.

1) $\mathcal{N}$ is a locally smooth manifold.

2) $\mathcal{N}$ is a complete metric space.

3) $\mathbb{E} \backslash\{(0,0)\}$ is a critical point of $\Phi$ if and only if $\mathbf{u}$ is a critical point of $\Phi$ constrained on $\mathcal{N}$.

4) $\Phi$ is bounded from below on $\mathcal{N}$.

Proof. 1) Differentiating expression (2.4) yields

$$
\Psi^{\prime}(\mathbf{u})[\mathbf{u}]=2\|\mathbf{u}\|^{2}-3 \int_{\mathbb{R}^{N}}\left(u^{3}+v^{3}\right)-8 \beta \int_{\mathbb{R}^{N}} u^{2} v^{2},
$$

and because of $\forall \mathbf{u} \in \mathcal{N}$, we have the fact that $\Psi(\mathbf{u})=0$.

Then, we obtain

$$
\Psi^{\prime}(\mathbf{u})[\mathbf{u}]=\Psi^{\prime}(\mathbf{u})[\mathbf{u}]-3 \Psi(\mathbf{u})=-\|\mathbf{u}\|^{2}-2 \beta \int_{\mathbb{R}^{N}} u^{2} v^{2}<0,
$$

Then, $\mathcal{N}$ is a locally smooth manifold near any point $\mathbf{u} \neq \mathbf{0}$ with $\Psi(\mathbf{u})=0$.

2) Let $\mathbf{u}_{n} \subset \mathcal{N}$ be a sequence such that $\left\|\mathbf{u}_{n}-\mathbf{u}_{0}\right\| \rightarrow 0$ as $n \rightarrow+\infty$. By the embedding theorem, we have $\left|u_{n}-u_{0}\right|_{p} \rightarrow 0$ and $\left|v_{n}-v_{0}\right|_{p} \rightarrow 0$ for $2 \leq p \leq 2^{*}$. It is clear that

$$
\begin{aligned}
& \Phi^{\prime}\left(\mathbf{u}_{n}\right)\left[\mathbf{u}_{n}\right]-\Phi^{\prime}\left(\mathbf{u}_{0}\right)\left[\mathbf{u}_{0}\right] \\
= & \int_{\mathbb{R}^{N}}\left|\Delta u_{n}\right|^{2}-\left|\Delta u_{0}\right|^{2}+\int_{\mathbb{R}^{N}} \lambda_{1}\left(u_{n}^{2}-u_{0}^{2}\right)+\int_{\mathbb{R}^{N}}\left|\Delta v_{n}\right|^{2}-\left|\Delta v_{0}\right|^{2}+\int_{\mathbb{R}^{N}} \lambda_{1}\left(v_{n}^{2}-v_{0}^{2}\right) \\
& -\int_{\mathbb{R}^{N}}\left(u_{n}^{3}-u_{0}^{3}\right)-\int_{\mathbb{R}^{N}}\left(v_{n}^{3}-v_{0}^{3}\right)-2 \beta \int_{\mathbb{R}^{N}}\left(u_{n}^{2} v_{n}^{2}-u_{0}^{2} v_{0}^{2}\right) .
\end{aligned}
$$

Since $\left|u_{n}-u_{0}\right|_{2} \rightarrow 0$ and $\left|v_{n}-v_{0}\right|_{2} \rightarrow 0$, applying Holder's inequality, we get

$$
\begin{aligned}
& \left|\int_{\mathbb{R}^{N}}\left(u_{n}^{2} v_{n}^{2}-u_{0}^{2} v_{0}^{2}\right)\right| \\
& \leq \int_{\mathbb{R}^{N}} u_{n}^{2}\left|v_{n}^{2}-v^{2}\right|+\int_{\mathbb{R}^{N}} v^{2}\left|u_{n}^{2}-u^{2}\right| \\
& \leq\left|u_{n}\right|_{4}^{2}\left(\int \mathbb{R}^{N}\left(v_{n}-v_{0}\right)^{2}\left(v_{n}-v_{0}\right)^{2}\right)^{\frac{1}{2}}+|v|_{4}^{2}\left(\int \mathbb{R}^{N}\left(u_{n}-u_{0}\right)^{2}\left(u_{n}-u_{0}\right)^{2}\right)^{\frac{1}{2}} \\
& \leq\left|u_{n}\right|_{4}^{2} \cdot\left|v_{n}-v_{0}\right|_{4} \cdot\left|v_{n}-v_{0}\right|_{4}+|v|_{4}^{2} \cdot\left|u_{n}-u_{0}\right|_{4} \cdot\left|u_{n}-u_{0}\right|_{4} \\
& \rightarrow 0,
\end{aligned}
$$

So we have $\Phi^{\prime}\left(\mathbf{u}_{n}\right)\left[\mathbf{u}_{n}\right]-\Phi^{\prime}\left(\mathbf{u}_{0}\right)\left[\mathbf{u}_{0}\right] \rightarrow 0$. Because of $\Phi^{\prime}\left(\mathbf{u}_{n}\right)\left[\mathbf{u}_{n}\right]=0$, we get $\Phi^{\prime}\left(\mathbf{u}_{0}\right)\left[\mathbf{u}_{0}\right]=0$. Using $\left\|\mathbf{u}_{n}\right\|^{2}>\rho$ and $\left\|\mathbf{u}_{n}-\mathbf{u}_{0}\right\| \rightarrow 0$, we get $\mathbf{u}_{n} \neq(0,0)$. Hence $\mathbf{u}_{n} \in \mathcal{N}$ and $\mathcal{N}$ is a complete metric space.

Taking the derivative of the functional $\Phi$ in the direction $\mathbf{h}=\left(h_{1}, h_{2}\right)$, we 
find

$$
\begin{aligned}
\Phi^{\prime}(\mathbf{u})[\mathbf{h}]= & \int_{\mathbb{R}^{N}}\left(\Delta u h_{1}+\lambda_{1} u h_{1}+\Delta v h_{2}+\lambda_{2} v h_{2}\right)-\int_{\mathbb{R}^{N}}\left(u^{2} h_{1}+v^{2} h_{2}\right) \\
& -\beta \int_{\mathbb{R}^{N}}\left(u v^{2} h_{1}+u^{2} v h_{2}\right) .
\end{aligned}
$$

The second derivative of $\Phi$ is given by

$$
\Phi^{\prime \prime}(\mathbf{u})[\mathbf{h}]^{2}=\|\mathbf{h}\|^{2}-2 \int_{\mathbb{R}^{N}}\left(u h_{1}^{2}+v h_{2}^{2}\right)-\beta \int_{\mathbb{R}^{N}}\left(u^{2} h_{2}^{2}+v^{2} h_{1}^{2}+4 u v h_{1} h_{2}\right) .
$$

So, we have

$$
\Phi^{\prime \prime}(\mathbf{0})[\mathbf{h}]^{2}=\|\mathbf{h}\|^{2},
$$

which is positive definite so that $\mathbf{0}$ is a strict minimum critical point of $\Phi$. As a consequence, we have that $\mathcal{N}$ is a smooth complete manifold, and there exists a constant $\rho>0$ such that

$$
\|\mathbf{u}\|^{2}>\rho, \quad \forall \mathbf{u} \in \mathcal{N} .
$$

3) Assume that $\left(u_{0}, v_{0}\right) \in \mathcal{N}$ is a critical point of $\Phi$ and with $\Psi(\mathbf{u})=\Phi^{\prime}(\mathbf{u})[\mathbf{u}]$, then there is a Lagrange multiplier $\wedge \in \mathbb{R}$ such that

$$
\Phi^{\prime}\left(u_{0}, v_{0}\right)=\wedge \Psi^{\prime}\left(u_{0}, v_{0}\right) \text {. }
$$

Apply both sides to $\left(u_{0}, v_{0}\right)$ and we can get

$$
0=\left(\Phi^{\prime}\left(u_{0}, v_{0}\right),\left(u_{0}, v_{0}\right)\right) \wedge\left(\Psi^{\prime}\left(u_{0}, v_{0}\right),\left(u_{0}, v_{0}\right)\right) .
$$

Combining (2.6) and (2.11), we get $\wedge=0$. Now (2.10) gives $\Phi^{\prime}\left(u_{0}, v_{0}\right)=0$, i.e. $\left(u_{0}, v_{0}\right)$, is a critical point of $\Phi$.

4) The functional constrained on $\mathcal{N}$ takes the form combining (2.3) and

$$
\Phi_{\mathcal{N}}(\mathbf{u})=\frac{1}{6}\|\mathbf{u}\|^{2}+\frac{1}{6} \beta \int_{\mathbb{R}^{N}} u^{2} v^{2},
$$

using (2.9) and (2.12), we can get

$$
\Phi(\mathbf{u}) \geq \frac{1}{6} \rho, \quad \forall \mathbf{u} \in \mathcal{N},
$$

So, $\Phi$ is bounded from below on $\mathcal{N}$.

Lemma 2.1. For every $\mathbf{u}=(u, v) \in \mathbb{E} \backslash\{(0,0)\}$, there is a number $t>0$ such that $t \mathbf{u} \in \mathcal{N}$.

Proof. For $(u, v) \in H^{2}\left(\mathbb{R}^{N}\right) \backslash\{(0,0)\}$ and $t>0$, we have

$$
\omega(t):=\Phi(t u, t v)=\frac{1}{2} t^{2}\|\mathbf{u}\|^{2}-\frac{1}{3} t^{3} \int_{\mathbb{R}^{N}}\left(u^{3}+v^{3}\right)-\frac{1}{2} \beta t^{4} \int_{\mathbb{R}^{N}} u^{2} v^{2} .
$$

On the one hand, we have $\omega(0)=0$ and $\omega(t) \geq C^{\prime} t^{2}$ for a small enough $t$. On the other hand, we have $\omega(t) \rightarrow-\infty$ as $t \rightarrow \infty$. So there is a maximum point $t_{m}$ of $t$. Moreover we get $\omega^{\prime}\left(t_{m}\right)=\Phi^{\prime}\left(t_{m} \mathbf{u}\right) \mathbf{u}=0$ and deduce $t_{m} \mathbf{u} \in \mathcal{N}$.

Lemma 2.2. Assume that $2 \leq N \leq 7$, then $\Phi$ satisfies the $P S$ condition constrained on $\mathcal{N}$.

Proof. Suppose $\mathbf{u}_{n}=\left(u_{n}, v_{n}\right) \in \mathcal{N}$ is a sequence i.e. 


$$
\Phi\left(\mathbf{u}_{n}\right) \rightarrow c \quad \text { and } \quad \Phi_{\mathcal{N}}^{\prime}\left(\mathbf{u}_{n}\right) \rightarrow 0, \quad \text { as } n \rightarrow \infty
$$

From (2.4) and (2.9), we can get $\mathbf{u}_{n}$ is bounded, then we have a weakly convergent subsequence $\mathbf{u}_{n} \rightarrow \mathbf{u}_{0} \in \mathbb{E}$ (for convenience denoted again by $\mathbf{u}_{n}$ ). Since $H$ is compactly embedded into $L^{P}\left(\mathbb{R}^{\mathbb{N}}\right)$ for $2 \leq N \leq 7$, we infer that

$$
\int_{\mathbb{R}^{N}} u^{3} \rightarrow \int_{\mathbb{R}^{N}} u_{0}^{3}, \int_{\mathbb{R}^{N}} v^{3} \rightarrow \int_{\mathbb{R}^{N}} v_{0}^{3}, \int_{\mathbb{R}^{N}} u^{2} \rightarrow \int_{\mathbb{R}^{N}} u_{0}^{2}, \int_{\mathbb{R}^{N}} u^{2} v^{2} \rightarrow \int_{\mathbb{R}^{N}} u_{0}^{2} v_{0}^{2} .
$$

Moreover, using the fact that $\mathbf{u}_{n} \in \mathcal{N}$ and (2.3), we have

$$
\|\mathbf{u}\|^{2}=\int_{\mathbb{R}^{N}}\left(u^{3}+v^{3}\right)+2 \beta \int_{\mathbb{R}^{N}} u^{2} v^{2} \rightarrow \int_{\mathbb{R}^{N}}\left(u_{0}^{3}+v_{0}^{3}\right)+2 \beta \int_{\mathbb{R}^{N}} u_{0}^{2} v_{0}^{2} \geq \rho .
$$

which implies that $\mathbf{u}_{0} \neq 0$. Letting

$$
\Phi_{\mathcal{N}}^{\prime}(\mathbf{u})=\Phi^{\prime}(\mathbf{u})-\eta \Psi^{\prime}(\mathbf{u}),
$$

denotes the constrained gradient of $\Phi$ on $\mathcal{N}$ with $\eta \in \mathbb{R}$. Taking the scalar product with $\mathbf{u}_{n} \neq 0$ and with $\Phi^{\prime}(\mathbf{u})[\mathbf{u}]=\Psi(\mathbf{u})=0$ we can get

$$
\eta_{n}\left(\Psi^{\prime}\left(\mathbf{u}_{n}, \mathbf{u}_{n}\right)\right) \rightarrow 0
$$

then, taking into account (2.6) and (2.7), we deduce that $\eta_{n}$ as $n \rightarrow 0$. We also have that $\left\|\Psi^{\prime}\left(\mathbf{u}_{n}\right)\right\|$ is bounded, so with (2.13) and the fact $\eta_{n}$ as $n \rightarrow 0$, we obtain

$$
\left\|\Phi^{\prime}\left(\mathbf{u}_{n}\right)\right\| \leq\left\|\nabla_{\mathcal{N}} \Phi\left(\mathbf{u}_{n}\right)\right\|+\left|\eta_{n}\right|\left\|\Psi^{\prime}\left(\mathbf{u}_{n}\right)\right\| \rightarrow 0, \text { as } n \rightarrow \infty .
$$

So we deduce that $\Psi^{\prime}\left(\mathbf{u}_{n}\right) \rightarrow 0$ To finish the proof, since $\Phi^{\prime}(\mathbf{u})\left[\mathbf{u}_{0}\right] \rightarrow 0$ as $n \rightarrow \infty$, it follows that $\mathbf{u}_{n} \rightarrow \mathbf{u}_{0} \in \mathbb{E}$ strongly.

Theorem 2.1. Suppose $\beta>\wedge^{+}$. The infimum of $\Phi$ on $\mathcal{N}$ is attained at some $\tilde{\mathbf{u}}$ with $\Phi(\tilde{\mathbf{u}})<\min \left\{\Phi\left(\mathbf{u}_{1}\right), \Phi\left(\mathbf{v}_{2}\right)\right\}$ and both components $\tilde{u}, \tilde{v} \neq 0$.

Theorem 2.2.

1) Let $\beta_{1}>0$ be the principal eigenvalue of

$$
\Delta^{2} \psi+\psi=\beta U_{1}^{2} \psi, \psi \in E,
$$

and let $\psi_{\beta_{1}}>0$ be the corresponding positive eigenfunction. Then there exists $\tau_{0}>0$ such that when $\beta \in\left(\beta_{1}-\tau_{0}, \beta_{1}\right)$, (1.3) has solutions $\left(u_{1 \beta}, v_{1 \beta}\right)$ of the form

$$
u_{1 \beta}=U_{1}+o\left(\beta_{1}-\beta\right) \text {, }
$$

and

$$
\begin{aligned}
v_{1 \beta} & =s \psi_{\beta_{1}}+o(s) \\
& =\frac{\beta-\beta_{1}}{\beta^{\prime}(0)} \psi_{\beta_{1}}+o\left(\beta_{1}-\beta\right) \\
& =\left(\beta_{1}-\beta\right) \frac{\int_{\mathbb{R}^{N}} U_{1} \psi_{\beta_{1}}^{2}}{\int_{\mathbb{R}^{N}} \psi_{\beta_{1}}^{3}} \psi_{\beta_{1}}+o\left(\beta_{1}-\beta\right)
\end{aligned}
$$

2). There exists $\tau_{1}>0$ such that when $\beta \in\left(-\tau_{1}, \tau_{1}\right)$, (1.3) has solutions $\left(u_{2 \beta}, v_{2 \beta}\right)$ of the form 


$$
\begin{aligned}
& u_{2 \beta}=U_{1}+\beta\left(\Delta^{2}+\lambda_{1}-2 U_{1}\right)^{-1}\left(U_{1} V_{2}^{2}\right)+o(\beta), \\
& v_{2 \beta}=V_{2}+\beta\left(\Delta^{2}+\lambda_{2}-2 V_{2}\right)^{-1}\left(U_{1}^{2} V_{2}\right)+o(\beta) .
\end{aligned}
$$

\section{Existence Results of Semi-Trivial Solutions and Non-Trivial Solutions}

System (1.5) admits two kinds of semi-trivial solutions of the form $(u, 0)$ and $(0, v)$. So we take $\mathbf{u}_{1}=\left(U_{1}, 0\right)$ and $\mathbf{v}_{2}=\left(0, V_{2}\right)$, where $U_{1}$ and $V_{2}$ are radially symmetric ground state solutions of the equation $\Delta^{2} w+\lambda_{i} w=w^{2}, i=1,2$ in $H^{2}\left(\mathbb{R}^{N}\right)$. Moreover, if we denote $w$ a radially symmetric ground state solution of (3.1)

$$
\Delta^{2} w+w=w^{2},
$$

then, by scaling, we can get

$$
U_{1}(x)=\lambda_{1} w\left(\sqrt[4]{\lambda_{1}} x\right), \quad V_{2}(x)=\lambda_{2} w\left(\sqrt[4]{\lambda_{2}} x\right),
$$

Hence, system (1.5) has two kinds of semi-trivial solutions $\mathbf{u}_{1}=\left(U_{1}, 0\right)$ and $\mathbf{v}_{2}=\left(0, V_{2}\right)$ with lowest energy among all the semi-trivial solutions.

\section{Definition 3.1.}

1) We define new Nehari manifold corresponding to the equations of (1.5) by

$$
\begin{aligned}
& \mathcal{N}_{1}=\left\{u \in H^{2}\left(\mathbb{R}^{N}\right) \backslash\{0\}: J_{1}(0)=0\right\}, \\
& \mathcal{N}_{2}=\left\{v \in H^{2}\left(\mathbb{R}^{N}\right) \backslash\{0\}: J_{2}(v)=0\right\},
\end{aligned}
$$

where

$$
J_{1}(u):=I_{1}^{\prime}(u)[u], J_{2}(v):=I_{2}^{\prime}(v)[v] .
$$

Let us define the tangent space to $\mathcal{N}$ on $\mathbf{u}_{1}$ and $\mathbf{v}_{2}$ by

$$
\begin{aligned}
& T_{\mathbf{u}_{1}} \mathcal{N}=\left\{\mathbf{h} \in H^{2} \times H^{2}: \Psi^{\prime}\left(\mathbf{u}_{1}\right)[\mathbf{h}]=0\right\}, \\
& T_{\mathbf{v}_{2}} \mathcal{N}=\left\{\mathbf{h} \in H^{2} \times H^{2}: \Psi^{\prime}\left(\mathbf{v}_{2}\right)[\mathbf{h}]=0\right\},
\end{aligned}
$$

And define the tangent space to $\mathcal{N}_{1}$ on $U_{1}$ and $\mathcal{N}_{2}$ on $V_{2}$ by

$$
\begin{aligned}
& T_{U_{1}} \mathcal{N}_{1}=\left\{h \in H^{2}\left(\mathbb{R}^{N}\right): J_{1}^{\prime}\left(U_{1}\right)[h]=0\right\}, \\
& T_{V_{2}} \mathcal{N}_{2}=\left\{h \in H^{2}\left(\mathbb{R}^{N}\right): J_{2}^{\prime}\left(V_{2}\right)[h]=0\right\} .
\end{aligned}
$$

We can prove the following equivalence:

$$
\mathbf{h}=\left(h_{1}, h_{2}\right) \in T_{\mathbf{u}_{1}} \mathcal{N} \Leftrightarrow h_{1} \in T_{U_{1}} \mathcal{N}_{1}, \quad \mathbf{h}=\left(h_{1}, h_{2}\right) \in T_{\mathbf{v}_{2}} \mathcal{N} \Leftrightarrow h_{2} \in T_{V_{2}} \mathcal{N}_{2} .
$$

If we denote by $D^{2} \Phi_{\mathcal{N}}$ the second derivative of $\Phi$ constrained on $\mathcal{N}$, using that $\mathbf{v}_{2}$ is a critical point of $\Phi$, plainly we obtain that

$$
D^{2} \Phi_{\mathcal{N}}\left(\mathbf{v}_{2}\right)[\mathbf{h}]^{2}=\Phi^{\prime \prime}\left(\mathbf{v}_{2}\right)[\mathbf{h}]^{2}, \quad \forall \mathbf{h} \in T_{\mathbf{v}_{2}} \mathcal{N} .
$$

2) We define the following Sobolev constants related to $U_{1}$ and $V_{2}$,

$$
S_{1}^{2}:=\inf _{\varphi \in E \backslash\{0\}} \frac{\|\varphi\|_{2}^{2}}{\int_{\mathbb{R}^{N}} U_{1}^{2} \varphi^{2}}, \quad S_{2}^{2}:=\inf _{\varphi \in E \backslash\{0\}} \frac{\|\varphi\|_{1}^{2}}{\int_{\mathbb{R}^{N}} V_{2}^{2} \varphi^{2}},
$$


and

$$
\wedge^{+}=\max \left\{S_{1}^{2}, S_{2}^{2}\right\}, \wedge^{-}=\min \left\{S_{1}^{2}, S_{2}^{2}\right\}
$$

\section{Proposition 3.1.}

1) If $\beta<\wedge^{-}$then $\mathbf{u}_{1}, \mathbf{v}_{2}$ is a strict local minimum of $\Phi$ constrained on $\mathcal{N}$.

2) If either $\beta>\wedge^{+}$, then $\mathbf{u}_{1}, \mathbf{v}_{2}$ is a saddle point of $\Phi$ constrained on $\mathcal{N}$. Moreover

$$
\inf _{\mathcal{N}} \Phi(\mathbf{u})<\min \left\{\Phi\left(\mathbf{u}_{1}\right), \Phi\left(\mathbf{v}_{2}\right)\right\}
$$

Proof. 1) Suppose $\beta<S_{2}^{2}$.

For $\mathbf{h} \in T_{\mathbf{v}_{2}} \mathcal{N}$ one has that

$$
D^{2} \Phi_{\mathcal{N}}\left(\mathbf{v}_{2}\right)[\mathbf{h}]^{2}=\Phi^{\prime \prime}\left(\mathbf{v}_{2}\right)[\mathbf{h}]^{2}=\left\|h_{1}\right\|_{1}^{2}+I_{2}^{\prime \prime}\left(V_{2}\right)\left[h_{2}\right]^{2}-\beta \int_{\mathbb{R}^{N}} V_{2}^{2} h_{1}^{2} .
$$

For one thing, since $\beta<S_{2}^{2}$ and the definition of $S_{2}^{2}$, there exists $c_{1}>0$ such that

$$
\left\|h_{1}\right\|_{1}^{2}-\beta \int_{\mathbb{R}^{N}} V_{2}^{2} h_{1}^{2} \geq c_{1}\left\|h_{1}\right\|_{1}^{2},
$$

For another thing, using (3.3) and the fact that $V_{2}$ is a minimum of $I_{2}$ on $\mathcal{N}_{2}$, there exists a constant $c_{2}>0$ such that

$$
I_{2}^{\prime \prime}\left(V_{2}\right)\left[h_{2}\right]^{2} \geq c_{2}\left\|h_{2}\right\|^{2} \text {. }
$$

Hence, using (3.6) and (3.7) we get

$$
D^{2} \Phi_{\mathcal{N}}\left(\mathbf{v}_{2}\right)[\mathbf{h}]^{2} \geq c_{1}\left\|h_{1}\right\|_{1}^{2}+c_{2}\left\|h_{2}\right\|^{2},
$$

proving that $V_{2}$ is a strict local minimum of $\Phi$ on $\mathcal{N}$.

When $\beta<S_{1}^{2}$, we can obtain the same result by using the same argument as above.

2) Assume $\beta>S_{2}^{2}$

In this case, we choose an element $\tilde{h}_{1} \in H^{2}\left(\mathbb{R}^{N}\right)$, such that

$$
S_{2}^{2}<\frac{\left\|\tilde{h}_{1}\right\|_{1}^{2}}{\int_{\mathbb{R}^{N}} \tilde{h}_{1}^{2} V_{2}^{2}}<\beta,
$$

Now, taking $\tilde{\mathbf{h}}_{1}=\left(\tilde{h}_{1}, 0\right) \in T_{\mathbf{v}_{2}} \mathcal{N}$ we get

$$
D^{2} \Phi_{\mathcal{N}}\left(\mathbf{v}_{2}\right)\left[\tilde{\mathbf{h}}_{1}\right]^{2}=\left\|\tilde{h}_{1}\right\|_{1}^{2}-\beta \int_{\mathbb{R}^{N}} V_{2}^{2} h_{1}^{2}<0,
$$

And taking $\tilde{\mathbf{h}}_{2}=\left(0, \tilde{h}_{2}\right) \in T_{\mathbf{v}_{2}} \mathcal{N}$ we get

$$
D^{2} \Phi_{\mathcal{N}}\left(\mathbf{v}_{2}\right)\left[\tilde{\mathbf{h}}_{2}\right]^{2}=I_{2}^{\prime \prime}\left(V_{2}\right)\left[\tilde{h}_{2}\right]^{2} \geq c_{2}\left\|h_{2}\right\|_{2}^{2} \text {. }
$$

Hence, $V_{2}$ is a saddle point of $\Phi$ on $\mathcal{N}$.

When $\beta>S_{1}^{2}$ we can obtain the same result using the same argument as above and obviously inequality (3.5) holds.

Next, we will give the proof of Theorem 2.1 and Theorem 2.2.

Proof. By the Ekelands variational principle [26], there exists a minimizing 
sequence $\mathbf{u}_{n} \in \mathcal{N}$, i.e.,

$$
\Phi\left(\mathbf{u}_{n}\right) \rightarrow c:=\inf _{\mathcal{N}} \Phi, \text { and } \Phi_{\mathcal{N}}^{\prime}\left(\mathbf{u}_{n}\right) \rightarrow 0
$$

Due to the Lemma 2.2, there exists $\tilde{\mathbf{u}} \in \mathcal{N}$ such that

$$
\mathbf{u}_{n} \rightarrow \overline{\mathbf{u}} \text { as } n \rightarrow \infty \text {. }
$$

so, $\overline{\mathbf{u}}$ is a minimum point of $\Phi$ on $\mathcal{N}$.

We have $(\bar{u}, \bar{v}) \in H^{2} \times H^{2}$. Then there exists $t>0$ such that $(t|\bar{u}|, t|\bar{v}|) \in \mathcal{N}$. So we get

$$
\|\overline{\mathbf{u}}\|^{2}=t \int_{\mathbb{R}^{N}}\left(|\bar{u}|^{3}+|\bar{v}|^{3}\right)+2 \beta t^{2} \int_{\mathbb{R}^{N}}|\bar{u}|^{2}|\bar{v}|^{2} .
$$

Combining

$$
\|\overline{\mathbf{u}}\|^{2}=\int_{\mathbb{R}^{N}}\left(|\bar{u}|^{3}+|\bar{v}|^{3}\right)+2 \beta \int_{\mathbb{R}^{N}}|\bar{u}|^{2}|\bar{v}|^{2},
$$

we get $0<t \leq 1$. According to the definition of $\Phi_{\mathcal{N}}$,

$$
\begin{aligned}
\Phi(t|\bar{u}|, t|\bar{v}|) & =\frac{1}{6} t^{2}\|\overline{\mathbf{u}}\|^{2}+\frac{1}{6} t^{4} \beta \int_{\mathbb{R}^{N}} \bar{u}^{2} \bar{v}^{2} \\
& \leq \frac{1}{6}\|\overline{\mathbf{u}}\|^{2}+\beta \int_{\mathbb{R}^{N}} \bar{u}^{2} \bar{v}^{2} \\
& =\Phi(\bar{u}, \bar{v})=c
\end{aligned}
$$

with $\Phi(t|\bar{u}|, t|\bar{v}|) \geq c$, we get $\left(u^{\prime}, v^{\prime}\right):=\Phi(t|\bar{u}|, t|\bar{v}|)$ is a nonnegative ground state solution of the system. We can conclude that both components of $\mathbf{u}^{\prime}$ are non-trivial. In fact, if the second component $v^{\prime} \equiv 0$, then $\mathbf{u}^{\prime}=\left(u^{\prime}, 0\right)$. So $\mathbf{u}^{\prime}=\left(u^{\prime}, 0\right)$ is the non-trivial solution of the system (1.5), Hence, we have

$$
I_{1}\left(u^{\prime}\right)=\Phi\left(\mathbf{u}^{\prime}\right)<\Phi\left(\mathbf{u}_{1}\right)=I_{1}\left(U_{1}\right) .
$$

However, this is a contradiction due to the fact that $U_{1}$ is a radial ground state solution of $\Delta^{2} u+\lambda u=u^{2}$. We conclude the first component $u^{\prime} \neq 0$ using the same way. Lastly, taking into account Proposition 3.1-(2) and $\beta>\wedge^{+}$we have

$$
\Phi\left(\mathbf{u}^{\prime}\right)<\min \left\{\Phi\left(\mathbf{u}_{1}\right), \Phi\left(\mathbf{v}_{2}\right)\right\} .
$$

\section{Bifurcation of Nontrivial Solutions}

In this subsection, we prove the existence of nontrivial solution of (1.5) by using local bifurcation theory (see [27]). The main results follow.

Proof. Consider the eigenvalue problem

$$
\Delta^{2} \psi+\psi=\beta U_{1}^{2} \psi, \psi \in E,
$$

It is well known that this problem admits a sequence of eigenvalues

$$
0<\beta_{1}<\beta_{2}<\cdots<\beta_{n}<\cdots .
$$

Moreover, we infer from [28] that the first eigenvalue $\beta_{1}>0$ is simple and the principle eigenfunction $\psi_{\beta_{1}}$ is a positive function. Set $\mathcal{S}^{*}=\left\{(\beta, u, v)=\left(\beta, U_{1}, 0\right)\right\}$, We shall consider the bifurcation of nontrivial solution of (1.5) from the semitrivial branch $\mathcal{S}^{*}$ near $\left(\beta, U_{1}, 0\right)$. To accomplish 
this, we apply the bifurcation results of Crandall and Rabinowitz. First, we define $F$ by

$$
F(\beta, u, v)=\left(\begin{array}{c}
\Delta^{2} u+\lambda_{1} u-u^{2}-\beta u v^{2} \\
\Delta^{2} v+\lambda_{2} v-v^{2}-\beta u^{2} v
\end{array}\right)
$$

Clearly, for $(\phi, \psi),\left(\phi_{1}, \psi_{1}\right),\left(\phi_{2}, \psi_{2}\right) \in E$, one sees that

$$
\begin{gathered}
F_{(u, v)}(\beta, u, v)[(\phi, \psi)]=\left(\begin{array}{c}
\Delta^{2} \phi+\lambda_{1} \phi-2 u \phi-\beta v^{2} \phi-2 \beta u v \psi \\
\Delta^{2} \psi+\lambda_{2} \psi-2 v \psi-2 \beta u v \phi-\beta u^{2} \psi
\end{array}\right) \\
F_{(u, v)(u, v)}(\beta, u, v)\left[\left(\phi_{1}, \psi_{1}\right)\left(\phi_{2}, \psi_{2}\right)\right]=\left(\begin{array}{c}
-2 \phi_{1} \phi_{2}-2 \beta \phi_{1} \psi_{2} v-2 \beta v \psi_{1} \phi_{2}-2 \beta u \psi_{1} \psi_{2} \\
-2 \psi_{1} \psi_{2}-2 \beta u \psi_{1} \phi_{2}-2 \beta v \phi_{1} \phi_{2}-2 \beta u \phi_{1} \psi_{2}
\end{array}\right) \\
F_{\beta}(\beta, u, v)=\left(\begin{array}{l}
-u v^{2} \\
-u^{2} v
\end{array}\right) \\
F_{\beta(u, v)}(\beta, u, v)[(\phi, \psi)]=\left(\begin{array}{l}
-v^{2} \phi-2 u v \psi \\
-u^{2} \psi-2 u v \phi
\end{array}\right)
\end{gathered}
$$

We define

$$
L(\phi, \psi)=F_{(u, v)}\left(\beta, U_{1}, 0\right)[(\phi, \psi)]=\left(\begin{array}{c}
\Delta^{2} \phi+\lambda_{1} \phi-2 U_{1} \phi \\
\Delta^{2} \psi+\lambda_{2} \psi-\beta U_{1}^{2} \psi
\end{array}\right)=\left(\begin{array}{c}
L_{1}(\phi) \\
L_{2}(\psi)
\end{array}\right)
$$

From (4.1) and (4.2), we get that the null space $N\left(L_{1}\right)=\operatorname{span}\left\{\psi_{\beta_{1}}\right\}$. The solution space of $L_{2}$ in $\mathbb{E}$ is $N_{1}=\operatorname{span}\left\{\frac{\partial U_{1}^{2}}{\partial x_{j}}: 2 \leq j \leq N\right\}$. Hence, the null space $N\left(L_{2}\right)$ is trivial. So the null space $N(L)=\operatorname{span}\left\{\left(\psi_{\beta_{1}}, 0\right)\right\}$, and $\psi_{\beta_{1}}$ is the principal eigenfunction of (4.1). The range space of $L$ is defined by

$$
R(L)=\left\{(f, g) \in E: \int_{\mathbb{N}^{N}} g \psi_{\beta_{1}}=0\right\} .
$$

Thus, $N(L)=\operatorname{codim} R(L)=1$. Since $\int_{\mathbb{R}^{N}} U_{1} \psi_{\beta_{1}}>0$, it follows from (5.6) that

$$
F_{\beta(u, v)}\left(\beta_{1}, U_{1}, 0\right)\left[\left(0, \psi_{\beta_{1}}\right)\right]=\left(\begin{array}{c}
0 \\
-U_{1}^{2} \psi_{\beta_{1}}
\end{array}\right) \neq R(L)
$$

Thus, we can apply the result of [27] to conclude that the set of positive solutions to $(1.5)$ near $\left(\beta_{1}, U_{1}, 0\right)$ is a smooth curve

$$
\Gamma=\left\{\left(\beta(s), u_{1 \beta}(s), v_{1 \beta}(s)\right): s \in\left(-\tau_{0}, \tau_{0}\right)\right\},
$$

such that $\beta(s)=\beta_{1}+\beta^{\prime}(0) s+o(s), \quad u_{1 \beta}(s)=U_{1}+o(s), \quad v_{1 \beta}(s)=s \psi_{\beta_{1}}+o(s)$, where $\tau_{0}>0$ is a small constant. Moreover, $\beta^{\prime}(0)$ can be calculated as (see, for example, [29] [30])

$$
\beta^{\prime}(0)=-\frac{\left\langle F_{(u, v)(u, v)}\left(\beta_{1}, U_{1}, 0\right)\left[\left(0, \psi_{\beta_{1}}\right)\left(0, \psi_{\beta_{1}}\right)\right], \mathcal{L}\right\rangle}{2\left\langle F_{\beta(u, v)}\left(\beta_{1}, U_{1}, 0\right)\left[\left(0, \psi_{\beta_{1}}\right)\right], \mathcal{L}\right\rangle}=-\frac{\int_{\mathbb{R}^{N}} \psi_{\beta_{1}}^{3}}{\int_{\mathbb{R}^{N}} U_{1} \psi_{\beta_{1}}^{2}},
$$

where $\mathcal{L}$ is a linear functional on $\mathbb{E}$ defined as $\langle(f, g), \mathcal{L}\rangle=\int_{\mathbb{R}^{N}} g \psi_{\beta_{1}}$. Hence, we infer from (4.7) and (4.8) that for $\beta_{1}-\tau_{0}<\beta<\beta_{1}$ 


$$
u_{1 \beta}=U_{1}+o\left(\beta_{1}-\beta\right)
$$

and

$$
\begin{aligned}
v_{1 \beta} & =s \psi_{\beta_{1}}+o(s) \\
& =\frac{\beta-\beta_{1}}{\beta^{\prime}(0)} \psi_{\beta_{1}}+o\left(\beta_{1}-\beta\right) \\
& =\left(\beta_{1}-\beta\right) \frac{\int_{\mathbb{R}^{N}} U_{1} \psi_{\beta_{1}}^{2}}{\int_{\mathbb{R}^{N}} \psi_{\beta_{1}}^{3}} \psi_{\beta_{1}}+o\left(\beta_{1}-\beta\right)
\end{aligned}
$$

Now, we give the proof of (2). As we know, $w_{0}=\left(U_{1}(x), V_{2}(x)\right)$ is the unique positive solution of (1.5) with $\beta=0$. Recalling the map defined in (4.3), we have

$$
F_{(u, v)}\left(0, U_{1}, V_{2}\right)[(\phi, \psi)]=\left(\begin{array}{c}
\Delta^{2} \phi+\lambda_{1} \phi-2 U_{1} \phi \\
\Delta^{2} \psi+\lambda_{2} \psi-2 V_{2} \psi
\end{array}\right)
$$

It is well known that $L_{3}=\Delta^{2}+\lambda_{1}-2 U_{1}$ and $L_{4}=\Delta^{2}+\lambda_{2}-2 V_{2}$ are both invertible; hence, $w_{0}$ is nondegenerate in $\mathrm{X} 2$ r, i.e., $F_{(u, v)}\left(0, U_{1}, V_{2}\right)$ exists. We infer from the implicit function theorem that there exists $\tau_{2}>0, R_{0}>0$ and $w_{2}(\beta):\left(-\tau_{2}, \tau_{2}\right) \rightarrow B_{R_{0}}\left(w_{0}\right)$ such that for any $\beta \in\left(-\tau_{2}, \tau_{2}\right)$, $F\left(\beta, w_{2}(\beta)\right)=F\left(\beta, u_{2 \beta}, v_{2 \beta}\right)=0$. Moreover, we can compute $(\phi, \psi)$. Differentiating $F\left(\beta, U_{1}, V_{2}\right)$ by $\beta$ at $\beta=0$, because of $F\left(0, U_{1}, V_{2}\right)=0$, we get

$$
F_{(u, v)}\left(0, U_{1}, V_{2}\right)[(\phi, \psi)]=\left(\begin{array}{c}
\Delta^{2} \phi+\lambda_{1} \phi-2 U_{1} \phi \\
\Delta^{2} \psi+\lambda_{2} \psi-2 V_{2} \psi-
\end{array}\right)=-\left(\begin{array}{c}
-U_{1} V_{2}^{2} \\
-U_{1}^{2} V_{2}
\end{array}\right)
$$

so

$$
\phi=\left(\Delta^{2}+\lambda_{1}-2 U_{1}\right)^{-1}\left(U_{1} V_{2}^{2}\right), \psi=\left(\Delta^{2}+\lambda_{2}-2 V_{2}\right)^{-1}\left(U_{1}^{2} V_{2}\right)
$$

This gives the expression of $\left(u_{2 \beta}, v_{2 \beta}\right)$.

$$
\begin{aligned}
& u_{2 \beta}=U_{1}+\beta\left(\Delta^{2}+\lambda_{1}-2 U_{1}\right)^{-1}\left(U_{1} V_{2}^{2}\right)+o(\beta), \\
& v_{2 \beta}=V_{2}+\beta\left(\Delta^{2}+\lambda_{2}-2 V_{2}\right)^{-1}\left(U_{1}^{2} V_{2}\right)+o(\beta) .
\end{aligned}
$$

\section{Summary}

In the paper, we studied the positive radial solutions for a higher order coupled system of Korteweg-de Vries equations in Theorem 2.1. Moreover, we proved the multiplicity of the equations by a bifurcation theory in Theorem 2.2.

\section{Conflicts of Interest}

The author declares no conflicts of interest regarding the publication of this paper.

\section{References}

[1] Gear, J. and Grimshaw, R. (1984) Weak and Strong Interactions between Internal Solitary Waves Equation. Studies in Applied Mathematics, 70, 235-258. https://doi.org/10.1002/sapm1984703235 
[2] Ablowitz, M., Kaup, D., Newell, A. and Segur, H. (1973) Nonlinear Evolution Equations of Physical Significance. Physical Review Letters, 31, 125-127. https://doi.org/10.1103/PhysRevLett.31.125

[3] Alarcon, E., Angulo, J. and Montenegro, J.F. (1999) Stability and Instability of Solitary Waves for a Nonlinear Dispersive System. Nonlinear Analysis, 36, 1015-1035. https://doi.org/10.1016/S0362-546X(97)00724-4

[4] Montenegro, J.F. (1995) Sistemas de Equações nao-lineares; Estudo local, global e estabilidade de ondas solitarias. Ph.D. Thesis, IMPA, Rio de Janeiro.

[5] Cui, S., Deng, D. and Tao, S. (2006) Global Existence of Solutions for the Cauchy Problem of the Kawahara Equation with L2 Initial Data. Acta Mathematica Sinica, 22, 1457-1466. https://doi.org/10.1007/s10114-005-0710-6

[6] Cui, S. and Tao, S. (2005) Strichartz Estimates for Dispersive Equations and Solvability of Cauchy Problems of the Kawahara Equation. Journal of Mathematical Analysis and Applications, 304, 683-702. https://doi.org/10.1016/j.jmaa.2004.09.049

[7] Doronin, G.G. and Larkin, N.A. (2007) Kawahara Equation in a Quarter-Plane and in a Finite Domain. Boletim da Sociedade Paranaense de Matemática, 3, 9-16.

[8] Doronin, G.G. and Larkin, N.A. (2008) Kawahara Equation in a Bounded Domain. Discrete \& Continuous Dynamical Systems-Series B, 10, 783-799. https://doi.org/10.3934/dcdsb.2008.10.783

[9] Faminskii, A.V. (2012) Weak Solutions to Initial-Boundary-Value Problems for Quasilinear Evolution Equations of an Odd Order. Advances in Difference Equations, 17, 421-470.

[10] Faminskii, A.V. and Larkin, N.A. (2010) Initial-Boundary Value Problems for Quasilinear Dispersive Equations Posed on a Bounded Interval. Electronic Journal of Differential Equations, 2010, 1-20.

[11] Gao, G. and Sun, S.M. (2016) A Korteweg-de Vries Type of Fifth-Order Equations on a Finite Domain with Point Dissipation. Journal of Mathematical Analysis and Applications, 438, 200-239. https://doi.org/10.1016/j.jmaa.2016.01.050

[12] Karpman, V.I. (1996) Stabilization of Soliton Instabilities by Higher-Order Dispersion: Fourth-Order Nonlinear Schrödinger-Type Equations. Physical Review E, 53, 1336-1339. https://doi.org/10.1103/PhysRevE.53.R1336

[13] Karpman, V.I. and Shagalov, A.G. (2000) Stability of Solitons Described by Nonlinear Schrödinger Type Equation with Higher-Order Dispersion. Physica D, 144, 194-210. https://doi.org/10.1016/S0167-2789(00)00078-6

[14] Kato, T. (2012) Well-Posedness for the Fifth Order KdV Equation. Funkcialaj Ekvacioj, 55, 17-53. https://doi.org/10.1619/fesi.55.17

[15] Larkin, N.A. and Simoes, M.H. (2013) General Boundary Conditions for the Kawahara Equation on Bounded Intervals. Electronic Journal of Differential Equations, 2013, 1-21. https://doi.org/10.1186/1687-1847-2013-1

[16] Larkin, N.A. and Simoes, M.H. (2015) The Kawahara Equation on Bounded Intervals and on a Half-Line. Nonlinear Analysis. Real World Applications, 127, 397-412. https://doi.org/10.1016/j.na.2015.07.008

[17] Panthee, M. and Scialom, M. (2008) On the Cauchy Problem for a Coupled System of KdV Equations: Critical Case. Advances in Difference Equations, 13, 1-26.

[18] Wang, H. and Cui, S. (2007) Well-Posedness of the Cauchy Problem of a Water Wave Equation with Low Regularity Initial Data. Mathematical and Computer Modelling, 45, 1118-1132. https://doi.org/10.1016/j.mcm.2006.10.001

[19] Wang, H., Cui, D. and Deng, D. (2007) Global Existence of Solutions for Kawahara 
Equation in Sobolev Spaces of Negative Indices. Acta Mathematica Sinica, 23, 1435-1446. https://doi.org/10.1007/s10114-007-0959-Z

[20] Yan, W. and Li, Y. (2010) The Cauchy Problem for Kawahara Equation in Sobolev Spaces with Low Regularity. Mathematical Methods in the Applied Sciences, 33, 1647-1660. https://doi.org/10.1002/mma.1273

[21] Yan, W. and Li, Y. (2011) Ill-Posedness of Kawahara Equation and Kaup-Kupershmidt Equation. Journal of Mathematical Analysis and Applications, 380, 486-492. https://doi.org/10.1016/j.jmaa.2011.03.047

[22] Zhao, X.Q. and Zhang, B.Y. (2019) Non-Homogeneous Boundary Value Problems of the Fifth-Order KdV Equation on a Bounded Interval. Journal of Mathematical Analysis and Applications, 470, 251-278. https://doi.org/10.1016/j.jmaa.2018.09.068

[23] Alvarez-Caudevilla, P., Colorado, E. and Fabelo, R. (2017) A Higher Order System of Some Coupled Nonlinear Schrödinger and Korteweg-de Vries Equations. Journal of Mathematical Physics, 58, Article ID: 111503. https://doi.org/10.1063/1.5010682

[24] Adams, R.A. and Fournier, J.F. (2003) Sobolev Spaces. Pure and Applied Mathematics. Academic Press, Cambridge.

[25] Lions, P.L. (1982) Symetrie et compacite dans les espaces de Sobolev. Journal of Functional Analysis, 49, 315-334. https://doi.org/10.1016/0022-1236(82)90072-6

[26] Ekeland, I. (1974) On the Variational Principle. Journal of Mathematical Analysis and Applications, 47, 324-353. https://doi.org/10.1016/0022-247X(74)90025-0

[27] Crandall, M.-G. and Rabinowitz, P.-H. (1971) Bifurcation from Simple Eigenvalues. Journal of Functional Analysis, 8, 321-340. https://doi.org/10.1016/0022-1236(71)90015-2

[28] Wang, J. and Shi, J.-P. (2017) Standing Waves of Coupled Schrodinger Equations with Quadratic Interactions from Raman Amplification in a Plasma.

[29] Du, Y.-H. and Shi, J.-P. (2007) Allee Effect and Bistability in a Spatially Heterogeneous Predator-Prey Model. Transactions of the American Mathematical Society, 359, 4557-4593. https://doi.org/10.1090/S0002-9947-07-04262-6

[30] Shi, J.-P. (1999) Persistence and Bifurcation of Degenerate Solutions. Journal of Functional Analysis, 169, 494-531. https://doi.org/10.1006/jfan.1999.3483 